\title{
No evidence of the planet orbiting the extremely metal-poor extragalactic star HIP 13044^
}

\author{
M. I. Jones ${ }^{1,2}$ and J. S. Jenkins ${ }^{2}$ \\ 1 Departamento de Astronomía y Astrofísica, Pontificia Universidad Católica de Chile, Av. Vicuña Mackenna 4860, $782-0436$ Macul, \\ Santiago, Chile \\ e-mail: mjones@aiuc.puc.cl \\ 2 Departamento de Astronomía, Universidad de Chile, Camino El Observatorio 1515, Las Condes, Casilla 36-D Santiago, Chile
}

Received 25 June 2013 / Accepted 25 August 2013

\begin{abstract}
Context. The recent discovery of three giant planets orbiting the extremely metal-poor stars HIP 11952 and HIP 13044 have challenged theoretical predictions of the core-accretion model. According to this, the metal content of the protoplanetary disk from which giant planets are formed is a key ingredient for the early formation of planetesimals prior to the runaway accretion of the surrounding gas.

Aims. We reanalyzed the original FEROS data that were used to detect the planets to prove or refute their existence, employing our new reduction and analysis methods.

Methods. We applied the cross-correlation technique to FEROS spectra to measure the radial velocity variation of HIP 13044 and HIP 11952. We reached a typical precision of $\sim 35 \mathrm{~m} \mathrm{~s}^{-1}$ for HIP 13044 and $\sim 25 \mathrm{~m} \mathrm{~s}^{-1}$ for HIP 11952, which is significantly superior to the uncertainties presented previously.

Results. We found no evidence of the planet orbiting the metal-poor extragalactic star HIP 13044. We show that given our radial velocity precision, and considering the large number of radial velocity epochs, the probability for a non-detection of the radial velocity signal recently claimed is lower than $10^{-4}$. Finally, we also confirm findings that the extremely metal-poor star HIP 11952 does not contain a system of two gas giant planets. These results reaffirm the expectations from the core-accretion model of planet formation.
\end{abstract}

Key words. planet-star interactions - planets and satellites: general - planetary systems

\section{Introduction}

During the past two decades more than 900 extrasolar planets have been detected ${ }^{1}$, with a few thousand more new candidates that have still to be confirmed (mainly from the Kepler mission). Although the planetary distribution has revealed a huge diversity of planetary properties, there are some strong correlations that give us clues about the formation scenarios and the dynamical evolution of these types of systems. In particular, one of the most important observational results is the planet-metallicity connection. This correlation shows a strong increase in the occurrence of giant planets toward more metal-rich stars (Gonzalez 1997; Santos et al. 2001; Fischer \& Valenti 2005). Moreover, only a few planets have been found around stars with $[\mathrm{Fe} / \mathrm{H}]<-0.5$. This observational result has been used in favor of the coreaccretion model of planet formation, where the abundance of metals in the protoplanetary disk is an important facet for the growth of cores in the disk, prior to the runaway gas accretion phase (e.g. Ida \& Lin 2004; Mordasini et al. 2012). The coreaccretion model is also supported in the low-mass regime, since there appears to be an under abundance of the lowest-mass rocky planets orbiting the most metal-rich stars (Jenkins et al. 2013a), arguing for the rapid growth of planetesimals towards the critical core-mass limit.

Recently, Setiawan et al. (2010; hereafter S10) and Setiawan et al. (2012; hereafter S12) announced the detection of

\footnotetext{
* Based on data obtained from the ESO Science Archive Facility.

1 http://exoplanet.eu
}

two planetary systems around the extremely metal-poor stars HIP 13044 and HIP 11952, respectively. The metallicities of these stars are both at the level of $[\mathrm{Fe} / \mathrm{H}] \sim-2 \mathrm{dex}$, which challenges one of the basic ingredients of the core-accretion model. The discovery of the planet orbiting HIP 13044 conjures up even more fascination, because this star is thought to be on the horizontal branch. Additionally, according to its kinematic motion, HIP 13044 is part of the Helmi Stream, meaning that it originated in a satellite galaxy of the Milky Way (Helmi et al. 1999; Chiba et al. 2000). Therefore, this would be the first extragalactic planet ever discovered, highlighting the universality of planet formation.

In this paper we report the results from a reanalysis of the original S10 and S12 FEROS datasets that were used to discover these planetary systems, along with additional observations, using a new method of radial velocity (RV) measurements that we have developed. Our results indicate that there is no evidence for these two planetary systems. Moreover, in the latter case, we confirm the results recently published by Desidera et al. (2013) and Müller et al. (2013), who have shown that there is no indication of giant planets orbiting HIP 11952.

\section{Observations and data reduction}

We used 46 spectra of HIP 13044 that were retrieved from the ESO archive. All of the data were taken with FEROS (Kaufer et al. 1999), between the end of 2009 and the beginning of 2011. The exposure times of the spectra were mostly $900 \mathrm{~s}$, with a 


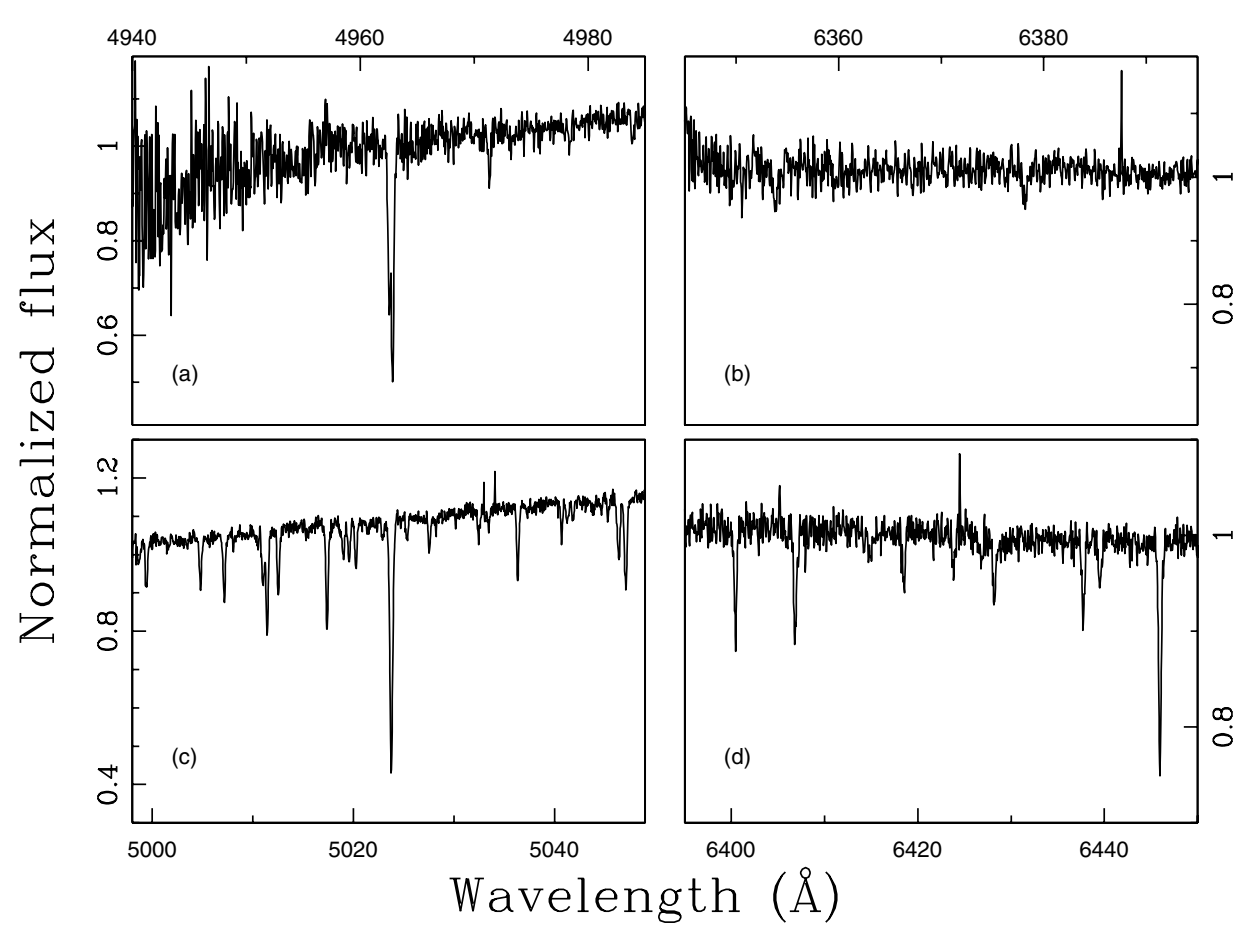

Fig. 1. Four typical chunks taken from one of the FEROS spectra of HIP 13044, showing chunks containing limited RV information (a) and b)) and chunks relatively rich in $\mathrm{RV}$ information (c) and $\mathbf{d})$ ). few of them with an exposure time of 1200 seconds. The typical signal-to-noise ratio (S/N) of the spectra is $\sim 80-100$ at $\sim 5500 \AA$. We used the FEROS Data Reduction System (DRS) to reduce the raw data. The FEROS pipeline performs a bias subtraction, flat-field normalization, order-tracing and orders extraction. The wavelength solution for every order was computed using two calibration lamps taken either during the afternoon prior to the observations, or during the morning, after the end of the observations. The wavelength solution leads to a typical RMS of $0.005 \AA$ from $\sim 900$ emission lines.

We also retrieved 72 FEROS spectra of the star HIP 11952 from the ESO Archive. The exposure times and typical S/N values are similar to those of the HIP 13044 dataset. The data reduction was performed in the same fashion as for HIP 13044.

\subsection{Radial velocity calculations}

The RVs were measured using a similar procedure as the one described in Jones et al. (2013), which has been tested for G and $\mathrm{K}$ dwarfs and giant stars. Based on this method, Jones et al. (2013) showed that it is possible to reach a long-term precision of $\sim 3 \mathrm{~m} \mathrm{~s}^{-1}$ using FEROS data. The main steps of this method are described below.

First, we computed the cross-correlation function (Tonry \& Davis 1979) between a template and the FEROS spectra. In this case, the templates correspond to one of the high $\mathrm{S} / \mathrm{N}$ observations of HIP 13044 and HIP 11952, instead of a numerical mask (e.g. Baranne et al. 1996; Pepe et al. 2002), even though numerical masks have proven their ability to generate highly precise RV measurements that can detect planets across a wide range of masses when coupled with highly stable instrumentation (e.g. Jenkins et al. 2009, 2013b; Mayor et al. 2009.) We applied this method to 100 chunks of $\sim 50 \AA$ width. These chunks were selected from 25 different orders, covering the region between $\sim 4000$ and $6500 \AA$. We then applied an iterative rejection method that removes every chunk velocity lying $2.5 \sigma$ away from the mean velocity. During this process many chunk velocities were rejected, mainly because of the lack of absorption lines in that chunk, low $\mathrm{S} / \mathrm{N}$ (especially in the blue edge of each order), and because of telluric lines (at $\sim 6000-6500 \AA$ ). Figure 1 shows four different chunks at different wavelengths. As can be seen, chunk (a) and (b) are noise dominated, and therefore do not contain enough information to measure reliable RVs via the cross-correlation method, whereas (c) and (d) do present several absorption lines, hence leading to reliable velocities. The stellar RV was obtained from the mean in the chunk velocities that were not excluded in the previous step. The error bars correspond to the error in the mean. In a similar way, we measured the velocity drift by cross-correlating the simultaneous lamp (from the sky fiber) with one of the lamps that was used to build the night wavelength calibration (from the sky fiber as well). The resulting RV shift was then subtracted from the measured stellar velocity. Finally, we applied the barycentric correction to the measured velocities, using the central time of the observation and the actual coordinates of the star, instead of the values recorded in the header (see details in Sect. 3). Figure 2 (upper panel) shows the RV variations computed for HIP 13044 using our method, which are listed in Table 1. The dotted line corresponds to the RV curve predicted by S10. The corresponding phase-folded diagram is also plotted (lower panel). The RMS to the S10 solution is $94 \mathrm{~m} \mathrm{~s}^{-1}$. Our error bars have typical values of $\sim 30-40 \mathrm{~m} \mathrm{~s}^{-1}$. The observed standard deviation of our RV measurements is $78 \mathrm{~m} \mathrm{~s}^{-1}$, which is larger than our instrumental errors. This probably can be explained by stellar jitter. In fact, HIP 13044 was classified as a horizontal branch star by $\mathrm{S} 10$, which are known to exhibit a high level of variability, mainly due to stellar oscillations (e.g. Setiawan et al. 2004; Hekker et al. 2006). The line profile asymmetry analysis presented in S10 (Fig. S2) reveals a scatter at the $\sim 100 \mathrm{~m} \mathrm{~s}^{-1}$ level, which agrees with our RV measurements. We computed a LombScargle (LS) periodogram (Scargle 1982) of our RV measurements, which is plotted in Fig. 3. The three horizontal lines from bottom to top correspond to false alarm probabilities of 0.01 , 0.001 and 0.0001 , respectively. As can be seen, there is no significant peak, in the range between 2 and 1000 days. This result is in stark contrast to the detection of a 16.2-day RV signal found by $\mathrm{S} 10$. 

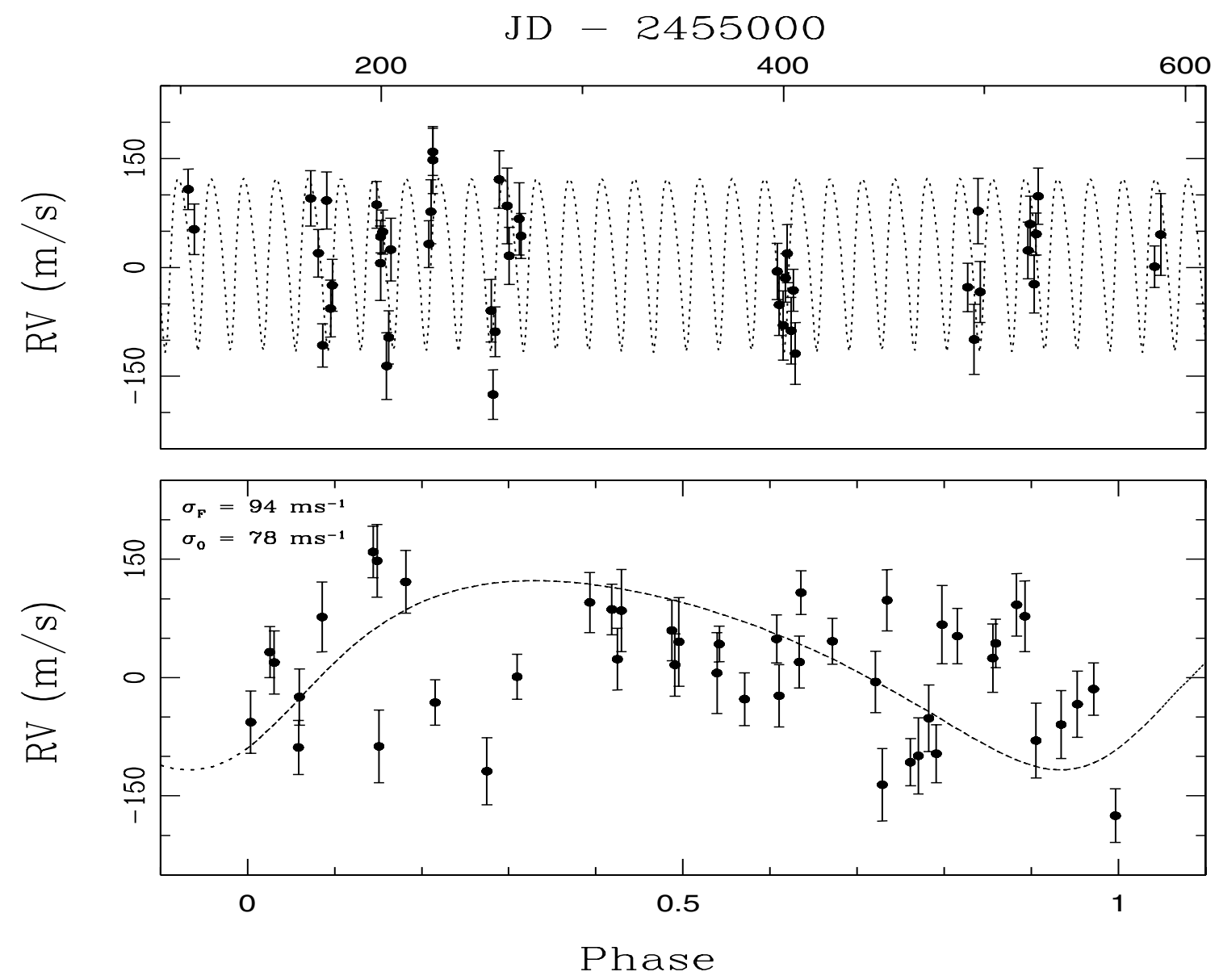

Fig. 2. Upper panel: 46 RV measurements of HIP 13044 (black dots). The dotted line corresponds to the RV curve predicted by S10. Lower panel: phase folded RVs to the orbital period predicted by S10. The RMS of the fit is $94 \mathrm{~m} \mathrm{~s}^{-1}$, whereas the standard deviation around zero is $78 \mathrm{~m} \mathrm{~s}^{-1}$.

To test the reliability of our results, we generated a synthetic dataset by computing the expected RV value at each observational epoch, using the orbital parameters derived by S10. We added to these velocities Gaussian-distributed noise with $\sigma$ equal to the observed standard deviation of our RV measurements $\left(78 \mathrm{~m} \mathrm{~s}^{-1}\right.$ ). Figure 4 shows the synthetic RVs (upper panel) and the corresponding LS periodogram (lower panel). It can be seen that even including Gaussian errors as large as $78 \mathrm{~m} \mathrm{~s}^{-1}$, it is possible to recover the original signal, since the highest peak in the periodogram corresponds exactly to the 16.2 days proposed by S10, and has a false alarm probability (FAP) of $\sim 10^{-4}$. This means that given the large number of data points (a total of 46 compared to only 36 measured by S10), we should be able to detect the 16.2-day RV signal claimed by S10, which is obviously not the case. This result forces us to conclude that the RV signal of 16.2 days claimed by S10 is not a real Doppler signal, hence there is no planet orbiting the extragalactic star HIP 13044 that has properties in agreement to those announced in S10.

\subsection{HARPS RVS}

As a secondary test of both the scatter in the RVs for HIP 13044 and the reality, or lack thereof, of the proposed planetary system, we measured 15 velocities using HARPS. We observed the star over 9 different nights, during four observing runs. We used the HARPS DRS to reduce the raw spectra and compute the wavelength solutions, but the procedure of computing the RVs could not be used on this star because no useable order-by-order cross-correlation functions could be generated. Therefore, we decided to use the HARPS-TERRA code (Anglada-Escude \& Butler 2012) to compute the RVs, which has been used to discover low-mass planets (e.g. Anglada-Escude et al. 2012). We obtained error bars between $5 \mathrm{~m} \mathrm{~s}^{-1}$ and $15 \mathrm{~m} \mathrm{~s}^{-1}$. Figure 5 (upper) shows the resulting RVs (corrected by a zero-point offset of $6.9 \mathrm{~m} \mathrm{~s}^{-1}$ ). The RV values are also listed in Table 2. The dashed line in Fig. 5 corresponds to the phase-folded signal predicted by S10. The lower panel shows the residuals to the S10 solution. It can be seen that the HARPS velocities and the S10 solution do not seem to be compatible. In particular there are two RV epochs that lie $\sim 140 \mathrm{~m} \mathrm{~s}^{-1}$ away from the predicted curve. Moreover, the RMS from a flat curve is $\sim 51 \mathrm{~m} \mathrm{~s}^{-1}$, which is smaller than the $\sim 58 \mathrm{~m} \mathrm{~s}^{-1}$ scatter around the S10 solution. Based on these results, we conclude that the HARPS data also argue against the reality of the planet predicted by S10. However, since the number of HARPS observations is very limited, especially because several spectra were taken within the same night or in consecutive nights, it is desirable to obtain new HARPS velocities in the future to confirm this result.

\section{Possible explanation of the RV discrepancy}

Although the reason for the discrepancy between S10 RV measurements and our velocities is not completely clear, we have some ideas of what might have caused the erroneous detection of the periodic RV signal in the S10 data.

First, we noted that the RV signal of 16.2 days claimed by $\mathrm{S} 10$ might be caused by the unfortunate combination of 
Table 1. FEROS RV measurements of HIP 13044.

\begin{tabular}{ccc}
\hline \hline JD - & RV & Error \\
2455000 & $\left(\mathrm{~m} \mathrm{~s}^{-1}\right)$ & $\left(\mathrm{m} \mathrm{s}^{-1}\right)$ \\
\hline 103.8958 & 107.7 & 27.8 \\
106.8039 & 52.6 & 34.9 \\
164.7661 & 95.3 & 38.2 \\
168.6638 & 19.7 & 32.7 \\
170.7306 & -107.3 & 29.8 \\
172.7060 & 92.3 & 39.6 \\
174.6620 & -56.5 & 39.3 \\
175.5667 & -24.5 & 35.7 \\
197.5781 & 86.5 & 32.1 \\
199.5313 & 5.9 & 51.2 \\
199.5742 & 42.5 & 22.5 \\
200.6403 & 49.0 & 30.4 \\
202.6077 & -135.8 & 46.0 \\
203.6124 & -96.4 & 36.8 \\
204.6604 & 24.6 & 43.3 \\
223.6162 & 32.3 & 32.3 \\
224.5880 & 76.8 & 44.2 \\
225.5371 & 159.3 & 32.4 \\
225.6111 & 148.1 & 46.1 \\
254.5321 & -59.6 & 43.1 \\
255.5427 & -175.1 & 34.0 \\
256.5481 & -88.5 & 34.2 \\
258.5431 & 121.3 & 39.8 \\
262.5545 & 84.9 & 52.1 \\
263.5521 & 16.1 & 39.5 \\
268.5183 & 67.1 & 49.7 \\
269.5178 & 43.4 & 30.8 \\
396.8810 & -5.5 & 38.9 \\
397.8721 & -51.5 & 42.3 \\
399.8659 & -79.8 & 47.6 \\
400.9363 & -14.5 & 33.3 \\
401.8983 & 19.2 & 40.0 \\
403.8446 & -87.2 & 46.0 \\
404.8909 & -31.6 & 29.0 \\
405.8531 & -118.7 & 42.6 \\
491.6448 & -27.3 & 33.4 \\
494.8776 & -99.2 & 48.4 \\
496.8584 & 77.7 & 44.9 \\
497.8354 & -33.7 & 41.9 \\
521.6808 & 23.4 & 39.0 \\
522.6921 & 59.8 & 38.4 \\
524.6873 & -23.0 & 39.5 \\
525.6789 & 46.1 & 28.9 \\
526.6963 & 98.1 & 38.7 \\
584.6150 & 1.1 & 28.7 \\
587.6262 & 45.4 & 56.3 \\
\hline & &
\end{tabular}

poor signal-to-noise ratio and the window function (sampling). Figure 6 shows the LS periodogram of the RV variations (upper panel) and the sampling (lower panel). In the upper panel we can clearly distinguish the 16.2-day peak, but in the lower panel, there is a peak at 31.4 days, which is very close to twice the 16.2-day period. Moreover, we noted that after removing the last two RV epochs in the S10 dataset it is possible to fit a 32.3-day orbit, which leads to almost the same RMS as the original fit (see upper panel of Fig. 7). Furthermore, we found that the new periodogram of the data shows a very strong peak at 32.3 days (lower panel in Fig. 7). These results strongly suggest that the RV signal claimed by $\mathrm{S} 10$ is related to the sampling instead of to a genuine Doppler signal in the data.

A second problem that we found in the S10 data is related to the barycentric correction. S10 mentioned that they used the FEROS DRS to produce 39 one-dimensional spectra, that were

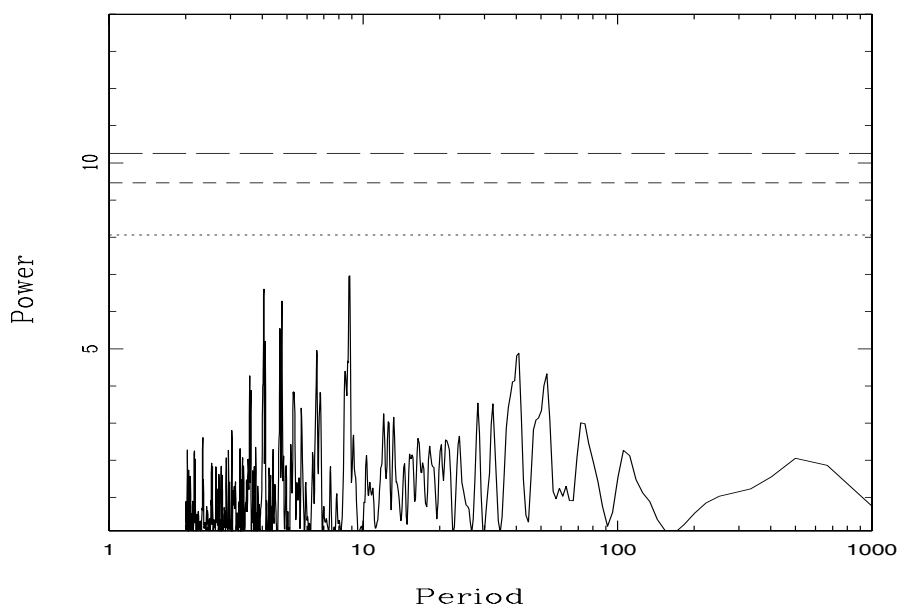

Fig. 3. LS periodogram of our RVs of HIP 13044. The three horizontal lines from bottom to top, correspond to a FAP of 0.01, 0.001 and 0.0001 , respectively.

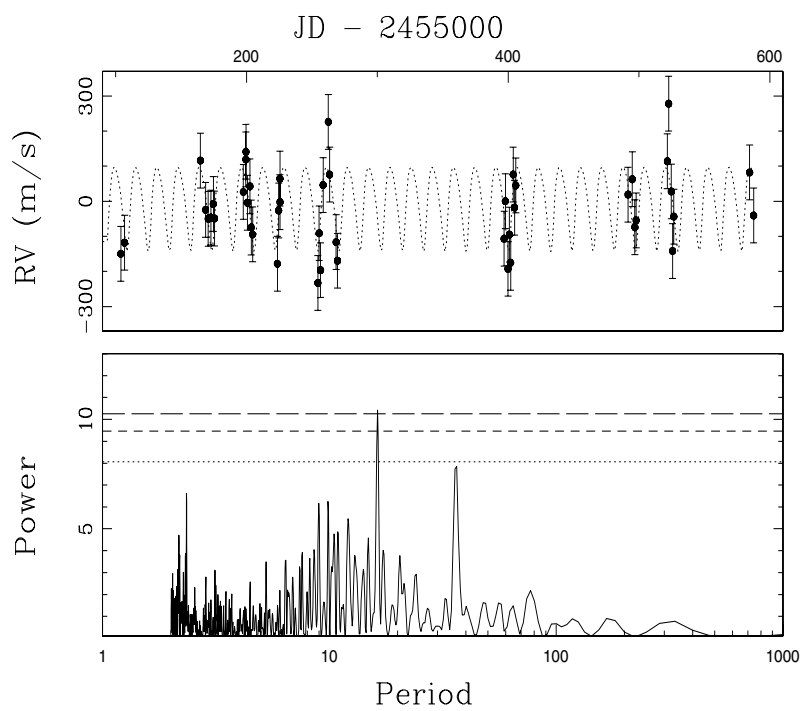

Fig. 4. Same as Fig. 2, but this time using a synthetic dataset, computed from the predicted RV amplitude at each epoch plus Gaussian noise with a standard deviation equal to $78 \mathrm{~m} \mathrm{~s}^{-1}$.

shifted to the rest frame after applying the barycentric velocity correction. However, this approach is not correct, since the FEROS-DRS barycentric correction introduces large systematic errors (see Müller et al. 2013). Additionally, the julian dates given by S10 correspond to the beginning of the observations instead of to the central time of the observation. For this specific dataset, this corresponds to a difference in time of $\sim 8-10 \mathrm{~min}$, leading to systematic errors in the barycentric velocity correction as large as $\sim 15 \mathrm{~m} \mathrm{~s}^{-1}$. These two errors, when combined, can produce systematic shifts in RV exceeding $20 \mathrm{~m} \mathrm{~s}^{-1}$.

Finally, we note that since there are large portions in most of the orders where there is a lack of absorption lines, the order-byorder cross-correlation can lead to inaccurate results. While S10 obtained typical error bars of $\sim 50-80 \mathrm{~m} \mathrm{~s}^{-1}$, we obtained uncertainties at the $\sim 30-40 \mathrm{~m} \mathrm{~s}^{-1}$ level. The reason why we obtained smaller error bars is because we computed the cross-correlation function in regions of $\sim 50 \AA$, rejecting chunks that lead to very deviant velocities. Figure 8 shows the difference between each chunk velocity and the median velocity from all chunks at each epoch (hence at each chunk number there are 46 velocities in 
M. I. Jones and J. S. Jenkins: No evidence of the planet HIP $13044 \mathrm{~b}$

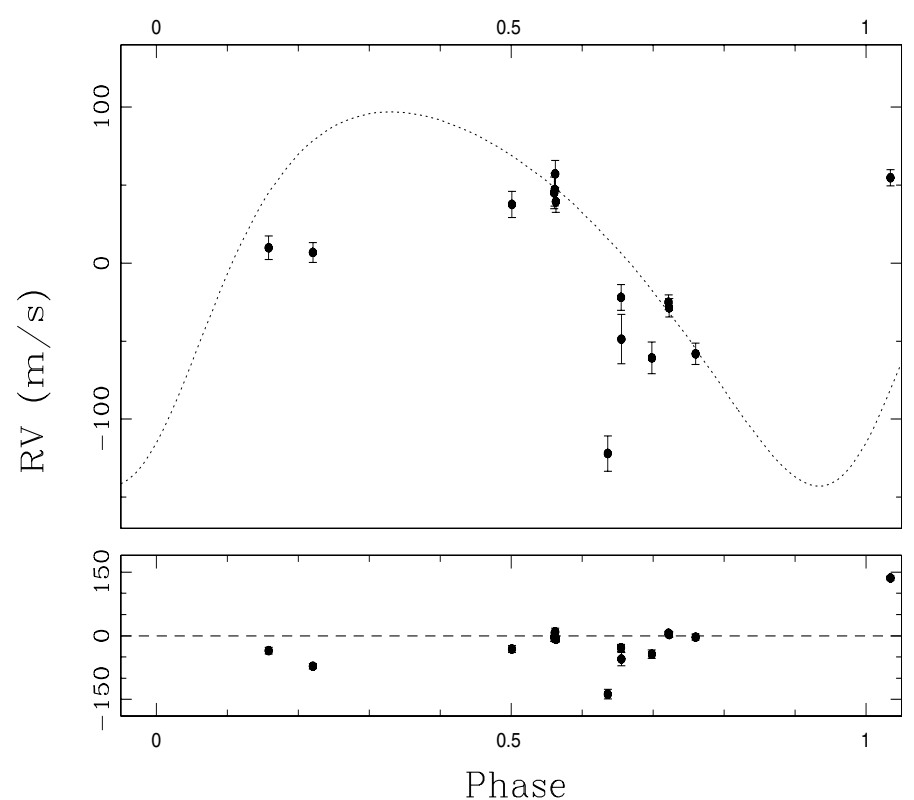

Fig. 5. HARPS RVs measured for HIP 13044 (black dots). The dashed line corresponds to the RV signal predicted by S10.

Table 2. HARPS RV measurements of HIP 13044.

\begin{tabular}{lcc}
\hline \hline $\begin{array}{l}\text { JD }- \\
2455000\end{array}$ & $\begin{array}{c}\mathrm{RV} \\
\left(\mathrm{m} \mathrm{s}^{-1}\right)\end{array}$ & $\begin{array}{c}\text { Error } \\
\left(\mathrm{m} \mathrm{s}^{-1}\right)\end{array}$ \\
\hline 1448.9294 & -128.9 & 11.3 \\
1449.9362 & -67.6 & 10.1 \\
1450.9352 & -65.0 & 6.8 \\
1462.9414 & 30.8 & 8.3 \\
1463.9067 & 38.2 & 10.1 \\
1463.9171 & 40.3 & 10.5 \\
1463.9299 & 50.4 & 8.6 \\
1463.9445 & 32.6 & 6.9 \\
1562.6326 & -28.8 & 8.2 \\
1562.6393 & -55.6 & 15.8 \\
1563.7164 & -31.9 & 4.7 \\
1563.7306 & -35.5 & 5.9 \\
1633.5839 & 47.9 & 5.3 \\
1635.5868 & 3.0 & 7.6 \\
1636.5963 & 0.0 & 6.3 \\
\hline
\end{tabular}

the $y$-axis direction). As can be seen, there are many chunks that produce deviant velocities at different epochs, which are rejected by our code (red crosses). On the other hand, chunks that lead to tighter velocities at different epochs are included in the final $\mathrm{RV}$ analysis (mostly corresponding to the black dots). It is worth mentioning that the chunks leading to constant velocities in time (black dots in Fig. 8) do not produce RVs close to zero, but they lead to RVs as high as $\sim 30 \mathrm{~km} \mathrm{~s}^{-1}$. Only after the barycentric velocity correction is applied, and the nightly drift is subtracted, do they become "flat". It is evident from Fig. 8 that including a complete order (compounded by four consecutive chunks) in the cross-correlation function produces a larger scatter in the final RVs.

\section{HIP11952}

As a part of the same RV program, S12 announced the detection of a planetary system around the extremely metal-poor $([\mathrm{Fe} / \mathrm{H}]=-1.9)$ star HIP 11952. As for HIP 13044, this discovery was very controversial since according to the core-accretion

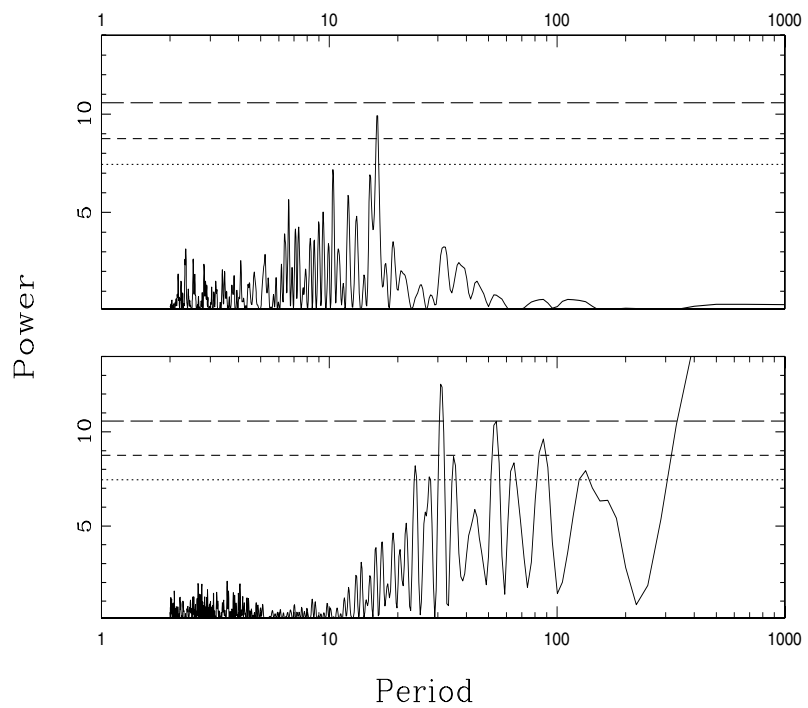

Fig. 6. LS periodogram of the S10 RVs (upper panel) and the sampling (lower panel). The three horizontal lines from bottom to top, correspond to a FAP of $0.01,0.001$ and 0.0001 , respectively.
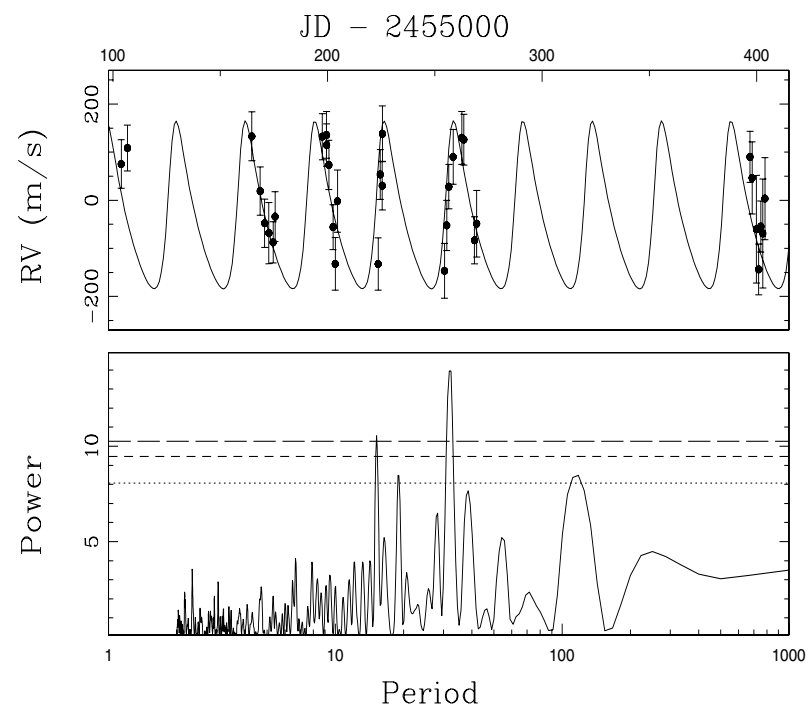

Fig. 7. Upper panel: Keplerian fit (solid line) of a 32.3-day orbit to the S10 data (black dots), excluding the last two RV epochs. The RMS to the fit is $55 \mathrm{~m} \mathrm{~s}^{-1}$. Lower panel: LS periodogram of the S10 data shown in the upper panel. The two highest peaks correspond to 15.2 days and 32.3 days. The three horizontal lines correspond from bottom to top to FAP of $0.01,0.001$ and 0.0001 , respectively.

model, giant planets are not expected to be formed around such metal-poor star, as we discussed above. However, based on much higher quality data taken with HARPS-N, Desidera et al. (2013) recently showed that there is no planetary system around HIP 11952 and that the RVs are flat, showing a small scatter at the $7 \mathrm{~m} \mathrm{~s}^{-1}$ level, which has also been confirmed by Müller et al. (2013).

To test this result and the reliability of our method, we also computed the RVs using 72 FEROS spectra of HIP 11952 in the same manner as explained above. Figure 9 shows the resulting RVs. The red cross corresponds to a deviant data point. As can be seen, the RV curve of HIP 11952 is flat, showing a scatter of only $28 \mathrm{~m} \mathrm{~s}^{-1}$, which can be explained solely by instrumental errors, since the mean value of the error bars is $27 \mathrm{~m} \mathrm{~s}^{-1}$, nearly twice as good as the S12 uncertainties on the same data. Clearly, 

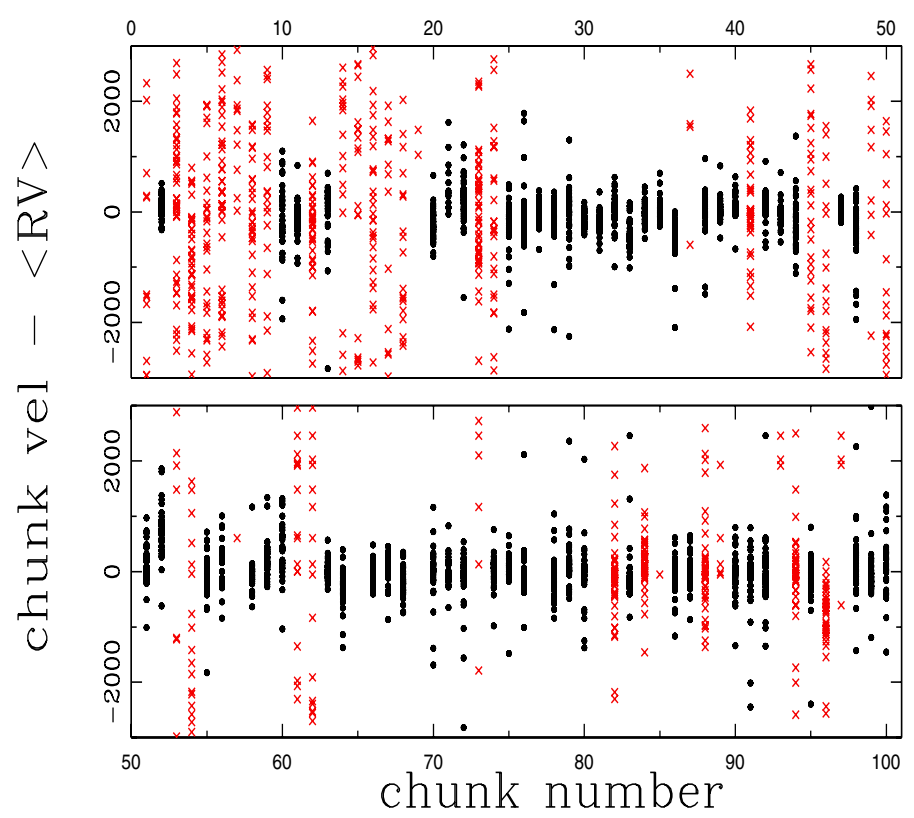

Fig. 8. Chunk velocities minus the median RV measured at different epochs. The red crosses show the chunks that lead to a large scatter. The black dots represent chunks that produce a lower dispersion in the RVs at different epochs, hence those that are included in the analysis.

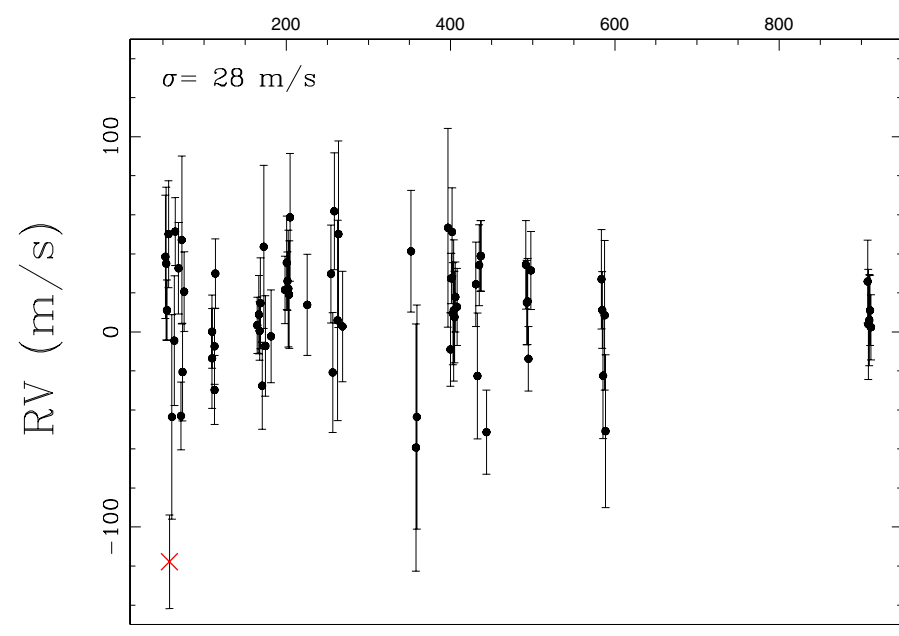

JD -2455000

Fig. 9. RV measurements of HIP 11952. The red cross corresponds to a deviant point that was excluded from the analysis. The error bars are typically $\sim 20-30 \mathrm{~m} \mathrm{~s}^{-1}$. The scatter around zero is $28 \mathrm{~m} \mathrm{~s}^{-1}$.

there is no indication of the two signals claimed by S12, both of them with predicted amplitudes above $100 \mathrm{~m} \mathrm{~s}^{-1}$. This result proves that our method leads to consistent RV measurements, even for extremely metal-deficient stars, reinforcing our results on HIP 13044, and it also confirms that HIP 11952 does not host a planetary system with the characteristics claimed by $\mathrm{S} 12$.

\section{Discussion}

Based on the RV analysis of 46 FEROS spectra, we found no evidence for a giant planet around HIP 13044, in contrast to the results announced by S10, who claimed the detection of a periodic RV signal that was interpreted to be caused by a giant planet with an orbital period of 16.2 days. We also confirm the flat RV time series for another extremely metal-poor star HIP 11952, which was claimed in S12 to have a system of two giant planets. These results again confirm the core-accretion scenario for planet formation, adding weight to the argument that it is difficult to form gas giant planets in dust-depleted protoplanetaryplanetary disks.

HIP13044 was classified as a horizontal branch star by S10, meaning that HIP $13044 b$ could have been a planet that had survived the common-envelope phase, since the radii of stars at the end of the red giant phase are much larger than the orbital distance of the proposed planet. In fact, no planets have yet been detected orbiting interior to $\sim 0.5 \mathrm{AU}$ around giant stars. Our results show that there is no need to develop complex models to explain the existence of this planet, and how it could have survived this disastrous phase of stellar evolution (Bear et al. 2011; Passy et al. 2012).

Based on the kinematic properties of HIP 13044, S10 highlighted that this star has an extragalactic origin, meaning the planet orbiting this star would have been the first planet known to originate from beyond the Milky Way galaxy. Our results state however, that there are still no planets around extremely metalpoor stars and that all of them have been formed around stars originating within our Galaxy.

Acknowledgements. M.J. acknowledges financial support from Fondecyt grant \#1120299 and ALMA-Conicyt grant \#31080027. J.J. acknowledges funding by Fondecyt through grant 3110004, the GEMINI-CONICYT FUND and from the Comité Mixto ESO-GOBIERNO DE CHILE. We also acknowledge support from Basal PFB-06 (CATA).

\section{References}

Anglada-Escude, G., \& Butler, R. P. 2012, ApJS, 200, 15

Anglada-Escude, G. Arriagada, P., Vogt, S. S., et al. 2012, ApJ, 751, 16

Baranne, A., Queloz, D., Mayor, M., et al. 1996, A\&A, 119, 373

Bear, E., Soker, N., \& Harpaz, A. 2011, ApJ, 733, 44

Chiba, M., \& Beers, T. 2000, AJ, 119, 2843

Desidera, S., Sozzetti, A., Bonomo, A. S., et al. 2013, A\&A, 554, A29

Hekker, S., Reffert, S., Quirrenbach, A., et al. 2006, A\&A, 454, 943

Fischer, D., \& Valenti, J. 2005, ApJ, 622, 1102

Gonzalez, G. 1997, MNRAS, 285, 403

Helmi, A., White, S. D. M., de Zeeuw, P. T., \& Zhao, H. 1999, Nature, 402, 53 Ida, S., \& Lin, D. 2004, ApJ, 604, 388

Jenkins, J. S., Jones, H. R. A., Goździewski, K., et al. 2009, MNRAS, 398, 911

Jenkins, J. S., Jones, H. R. A., Tuomi, M., et al. 2013a, ApJ, 766, 67

Jenkins, J. S., Tuomi, M., Brasser, R., Ivanyuk, O., \& Murgas, F. 2013b, ApJ, 771,41

Jones, M. I., Jenkins, J. S., Rojo, P., Melo, C. H. F., \& Bluhm, P. 2013, A\&A, 556, A78

Kaufer, A., Stahl, O., Tubbesing, S., et al. 1999, The Messenger, 95, 8

Mayor, M., Udry, S., Lovis, C., et al. 2009, A\&A, 493, 639

Mordasini, C., Alibert, Y., Benz, W., Klahr, H., \& Henning, T. 2012, A\&A, 541, A97

Müller, A., Roccatagliata, V., Henning, T., et al. 2013, A\&A, 556, A3

Passy, J-C., Mac Low, M-M., \& De Marco O. 2012, ApJ, 759, 30

Pepe, F., Mayor, M., Galland, F., et al. 2002, A\&A, 388, 632

Santos, N. C., Israelian, G., \& Mayor, M. 2001, A\&A, 373, 1019

Scargle, J. D. 1982, ApJ, 263, 835

Setiawan, J., Pasquini, L., da Silva, L., et al. 2004, A\&A, 421, 241

Setiawan, J., Klement, R. J., Henning, T., et al. 2010, Science, 330, 1642

Setiawan, J., Roccatagliata, V., Fedele, D., et al. 2012, A\&A, 540, A141

Tonry, J., \& Davis, M. 1979, AJ, 84, 1511 